Measur ement and Est i mat i on of the Parti cl e Si ze Di stri but i on by the Buoyancy Wei ghi ng- Bar Met hod and the Rosi $n$ - Ramm er Equat i on

\begin{tabular}{|l|l|}
\hline 著者 & $\begin{array}{l}\text { TAMBUN Rondang, FURUKAWA Kat suhi ko, H RAYAMA } \\
\text { Nasayuki, SHI MAZU Masami t su, YAMANAKA Shi nya, } \\
\text { OH RA Yui chi }\end{array}$ \\
\hline $\begin{array}{l}\text { j our nal or } \\
\text { publ i cat i on t i t l e }\end{array}$ & JOURNAL OF CHEM CAL ENG NEERI NG OF JAPAN \\
\hline vol une & 49 \\
\hline number & 2 \\
\hline page r ange & $229-233$ \\
\hline year & $2016-02-20$ \\
\hline URL & ht t p: //hdl . handl e. net /10258/00009944 \\
\hline
\end{tabular}




\title{
Measurement and Estimation of the Particle Size Distribution by the Buoyancy Weighing-Bar Method and the Rosin-Rammler Equation
}

\author{
Rondang TAMBUN ${ }^{1,2}$, Katsuhiko FURUKAWA ${ }^{3}$, Masayuki HIRAYAMA ${ }^{3}$, \\ Masamitsu SHIMADZU ${ }^{4}$, Shin-ya YAMANAKA ${ }^{4}$ and Yuichi OHIRA ${ }^{4}$
}

\author{
${ }^{1}$ Department of Chemical Engineering, University of Sumatera Utara, Jl. Almamater Kampus \\ USU Medan 20155, Indonesia \\ ${ }^{2}$ Division of Chemical and Materials Engineering, Graduate School of Engineering, \\ Muroran Institute of Technology, Muroran, Hokkaido 050-8585, Japan \\ ${ }^{3}$ Taihoku Civil Engineering and Construction Co., Furano, Hokkaido 076-0031, Japan \\ ${ }^{4}$ Division of Sustainable and Environmental Engineering, Graduate School of Engineering, \\ Muroran Institute of Technology, Muroran, Hokkaido 050-8585, Japan
}

\begin{abstract}
Keywords: Particle Engineering, Particle Size Distribution, Buoyancy, Rosin-Rammler Equation, Fine Particle

Measuring the size distribution of fine particles $(<5 \mu \mathrm{m})$ in a few hours is difficult when using the sedimentation method due to a decrease in sedimentation rate. Herein, we discussed the validity of using a combination of the buoyancy weighing-bar method and the Rosin-Rammler equation to estimate the particle size distribution. When the cumulative mass oversize exceeds about 0.15 , the Rosin-Rammler equation can be used to estimate the particle size distributions of suspended solids.
\end{abstract}

\section{Introduction}

To remove suspended solids by the sedimentation method, information about the particle size distribution is important. Typically, the particle size distribution is measured by the Andreasen pipette method (Andreasen, 1928), a hydrometer (Bouyoucos, 1927), the centrifugal sedimentation method (Donoghue and Bostock, 1955), microscopy (Stober and Fink, 1968), laser diffraction/scattering method (Minoshima et al., 2005), or the Coulter counter method (Coulter, 1953). Although the equipment is economical, the Andreasen pipette, microscopy, and the hydrometer methods are difficult to perform and time consuming. In contrast, the other methods quickly provide highly accurate results, but require expensive equipment. Hence, a reliable and affordable method to measure the particle size distribution in a water treatment plant does not exist.

Our research aims to develop a method to measure the particle size distribution using the buoyancy weighing-bar method. We have reported that the particle size distribution of settling particles can be measured using the buoyancy weighing-bar method (Obata et al., 2009; Furukawa et al., 2010; Ohira et al., 2010). In this method, a change in density due to settling particles is measured by weighing buoyancy against a weighing bar hung in the suspension. Then, the particle size distribution is calculated using the length of the weighing bar and the change over time in the apparent mass of the weighing bar (Obata et al., 2009). This method is very economical as the measuring apparatus consist of an analytical balance, a personal computer, and a weighing bar. Moreover, the measurement results are almost identical to the sedimentation balance method (Odén, 1925). However, particles less than about $5 \mu \mathrm{m}$ cannot be measured directly.
To control the sedimentation process of suspended solids from a water treatment plant, the particle size distributions of particles less than $5 \mu \mathrm{m}$ must be determined. Generally, log-normal or Rosin-Rammler distribution is often used for expression of particle size distribution (Miwa, 1981; Hidaka and Kamiya, 2014). Although the log-normal distribution has some advantages in terms of mathematical analysis, it is confined within relatively narrow particle size distribution (Miwa, 1981). Thus, we now demonstrate the buoyancy weighing-bar method and the Rosin-Rammler equation (Allen, 1990) could be applied to estimation of particle size distribution containing fine particles. In this paper, we discuss the estimation validity of the particle size distribution using the Rosin-Rammler equation.

\section{Theory}

Here, we briefly describe the theory of the buoyancy weighing-bar method because it is described in detail elsewhere (Obata et al., 2009; Furukawa et al., 2010; Ohira et al., 2010). Equation (1) shows the buoyant mass balance for a weighing bar in a suspension.

$$
\begin{aligned}
W_{0}-W=\left(W_{0}-W_{\infty}\right) \int_{x_{i}}^{x_{\max }} f(x) \mathrm{d} x \\
+\left(W_{0}-W_{\infty}\right) \int_{x_{\min }}^{x_{i}} \frac{v(x) t}{h} f(x) \mathrm{d} x,
\end{aligned}
$$

Where, $W$ is the buoyant mass, $h$ is the submerged length of the weighing bar, $v(x)$ is the settling velocity of the particle, and $f(x)$ is the mass frequency with particle size $x$. The apparent mass of weighing bar $G$ in the suspension is given by Eq. (2).

$$
G=V_{\mathrm{B}} \rho_{\mathrm{B}}-W=V_{\mathrm{B}}\left(\rho_{\mathrm{B}}-\rho_{\mathrm{S}}\right),
$$


Where, $\rho_{\mathrm{S}}$ is the suspension density, $\rho_{\mathrm{B}}$ is the weighing bar density in the suspension, and $V_{\mathrm{B}}$ is the weighing bar volume. Differentiating Eqs. (1) and (2) with respect to time $t$ provides Eq. (3).

$$
G=G_{\mathrm{R}}+\left(\frac{\mathrm{d} G}{\mathrm{~d} t}\right) t .
$$

The value of $G_{\mathrm{R}}$ is calculated from the tangent line based on Eq. (3). The cumulative mass oversize is given by Eq. (4).

$$
R=\int_{x}^{x_{\max }} f(x) \mathrm{d} x=\frac{G_{\mathrm{R}}-G_{0}}{G_{\infty}-G_{0}} .
$$

Particle size $x$ is given by the following equation using the Stokes formula.

$$
x=\sqrt{\frac{18 \mu_{\mathrm{L}} v(x)}{g\left(\rho_{\mathrm{P}}-\rho_{\mathrm{L}}\right)}}=\sqrt{\frac{18 \mu_{\mathrm{L}} h}{g\left(\rho_{\mathrm{P}}-\rho_{\mathrm{L}}\right) t}},
$$

Where, $g$ is the gravitational acceleration and $\mu_{\mathrm{L}}$ is the viscosity of the dispersion liquid. The particle size distribution of the suspended particles is calculated using the particle size $x$ at each time $t$, and subsequently plotting the cumulative mass oversize $R$.

The Rosin-Rammler equation describes the relationship between particle size $x$ and cumulative mass oversize $R$ (Allen, 1990). The cumulative mass oversize $R$ can be expressed as a function of particle size $x$ as Eq. (6).

$$
R=\exp \left[-\left(\frac{x}{x_{e}}\right)^{n}\right]
$$

Where, $x_{\mathrm{e}}$ is the absolute size constant and $n$ is the distribution constant. To determine these parameters, Eq. (6) can be rewritten as Eq. (7).

$$
\ln \ln \left(\frac{1}{R}\right)=n \ln \left(\frac{x}{x_{e}}\right)=n \ln x-n \ln x_{e} .
$$

Graphing $\ln \ln (1 / R)$ versus $\ln x$ can determine the distribution constant $n$ from the slope, while the slope and intercept can determine the absolute size constant $x_{\mathrm{e}}$. The particle size distribution can be calculated using Eq. (6), the distribution constant, and the absolute size constant.

\section{Materials and Methods}

Schematic diagrams of the experimental apparatus and the weighing bar are reported elsewhere (Obata et al., 2009; Furukawa et al., 2010; Ohira et al., 2010). The experimental apparatus consisted of an analytical balance, personal computer, graduated cylinder, weighing bar, etc. The weighing bar was an aluminum slit-cylinder (O.D.: $40 \mathrm{~mm}$, I.D.: $30 \mathrm{~mm}$, cylinder length: $210 \mathrm{~mm}$, slit depth: $5.0 \mathrm{~mm}$, slit length: $200 \mathrm{~mm}$, submerged length: 200 $\mathrm{mm}$ ), and according to JIS Z8807 (1976), its density was $2.70 \times 10^{3}$ $\mathrm{kg} \cdot \mathrm{m}^{-3}$. The graduated cylinder (diameter: $65 \mathrm{~mm}$, Sanplatec Co., Ltd.) was 1 L. An analytical balance (GR-300, A\&D Co., Ltd., minimum readout mass $0.1 \mathrm{mg}$ ) had a below-balance-weighing hook for hanging measurement, and was equipped with an RS-232C interface, which was used to connect the balance with a personal computer. Data were collected in $3 \mathrm{~s}$ intervals. To avoid external effects such as airflow and temperature changes, the experimental apparatus was placed in an insulated vessel with a heater connected to a thermostat to maintain a constant temperature of $298 \mathrm{~K}$. Additionally, a different principle, the laser diffraction/scattering (MT3000EX, Nikkiso Co., Ltd.), was used to measure the particle size distribution.

JIS Test Powders 1, class 3 (median size: 6.6-8.6 $\mu \mathrm{m}$, Silica sand), class 10 (median size: 4.8-5.7 $\mu \mathrm{m}$, Flyash), class 11 (median size: 1.6-2.3 $\mu \mathrm{m}$, KANTO (Japanese) loam), and class 17 (median size: 1.9-2.4 $\mu \mathrm{m}$, Calcium carbonate, heavy) were used as authorization particles (JIS Z8901, 2006). Sodium hexametaphosphate (NaHMP, Kanto Chemical Co., Inc.) with a concentration of $10 \mathrm{~kg} \cdot \mathrm{m}^{-3}$ and a NaHMP solution with a concentration of $1.0 \mathrm{~kg} \cdot \mathrm{m}^{-3}$ were used as the dispersant and dispersion liquid, respectively. The particle densities measured by the pycnometer (JIS Z8807, 1976) were $2.61 \times 10^{3} \mathrm{~kg} \cdot \mathrm{m}^{-3}$ (class 3), $2.14 \times 10^{3} \mathrm{~kg} \cdot \mathrm{m}^{-3}$ (class 10), $3.00 \times 10^{3} \mathrm{~kg} \cdot \mathrm{m}^{-3}$ (class 11), and $2.80 \times 10^{3} \mathrm{~kg} \cdot \mathrm{m}^{-3}$ (class17). The density and viscosity of the NaHMP solution measured by the pycnometer (JIS Z8807, 1976) and Ubbelohde viscometer (JIS K8803-01, 2011) were $1.00 \times 10^{3}$ $\mathrm{kg} \cdot \mathrm{m}^{-3}$ and $0.89 \mathrm{mPa} \cdot \mathrm{s}$, respectively.

About $2 \mathrm{~L}$ of seepage water at the construction site near the Sorachi River in Furano, Hokkaido, Japan was obtained as a natural suspension. The suspended solid concentration, ignition loss and solid density were analyzed according to JIS K0102 (2008) and JIS A1202 (2009). The concentration of suspended solids in the seepage water was $7.8 \mathrm{~kg} \cdot \mathrm{m}^{-3}$, while the density and ignition loss of the suspended solids were $2.71 \times 10^{3} \mathrm{~kg} \cdot \mathrm{m}^{-3}$ and 0.041 , respectively. The concentration of NaHMP added as a dispersant, was $0.78 \mathrm{~kg}$ • $\mathrm{m}^{-3}$.

To prepare a suspension, $1 \mathrm{~L}$ of the dispersion liquid and the particles to be tested were mixed in a graduated cylinder. A weighing bar was hung from the analytical balance via a hanging wire, which did not extend due to the weight of the weighing bar. After thoroughly stirring the suspension, the weighing bar was set in the suspension, and this was recorded as $0 \mathrm{~s}$. Data, which consisted of time and the corresponding apparent mass of the submerged weighing bar, were recorded on a personal computer. Then, the particle size distribution was determined and estimated based on the aforementioned theory.

\section{Results and Discussion}

\subsection{Combination validity of the Rosin-Rammler equation}

The particle size distributions of JIS Test Powders 1 , class 3 (Silica sand) measured by the buoyancy weighing-bar method within $2 \mathrm{~h}$ are reported in a previous paper (Obata et al., 2009). The minimum particle size that can be measured in the NaHMP solution is about $5 \mu \mathrm{m}$. To estimate particle sizes less than $5 \mu \mathrm{m}$, the absolute size constant $x_{\mathrm{e}}$ and distribution constant $n$ in the Rosin-Rammler equation are calculated using previous data (Obata et al., 2009).

Figure 1 shows the Rosin-Rammler plot. Because the slope and intercept are 0.883 and -2.24 , the absolute size constant $x_{\mathrm{e}}$ and distribution constant $n$ are $12.6 \mu \mathrm{m}$ and 0.883, respectively. Figure 2 shows the particle size distributions of JIS Test Powders 1, class 3 (Silica sand). The open circles and dashed line denote previous data (Obata et al., 2009) and the particle size distribution calculated by the Rosin-Rammler equation, respectively. The dashed line estimates that the cumulative mass oversize $R$ at $1 \mu \mathrm{m}$ is about 0.9 . The filled circles in Figure 2 show the standard data for the particle size by the sedimentation balance method from Japan Industrial Standard (JIS), while the squares denote the particle size distribution measured within $24 \mathrm{~h}$. The particle size distribution calculated by the Rosin-Rammler equation is close to the standard data. Additionally, the particle size distribution measured within 24 $\mathrm{h}$ agrees well with that calculated by the Rosin-Rammler equation. Hence, we conclude that the Rosin-Rammler equation and data measured within 2 h by the buoyancy weighing-bar method can estimate the particle size distribution.

\subsection{Estimation accuracy}

The Rosin-Rammler equation and two data points can 


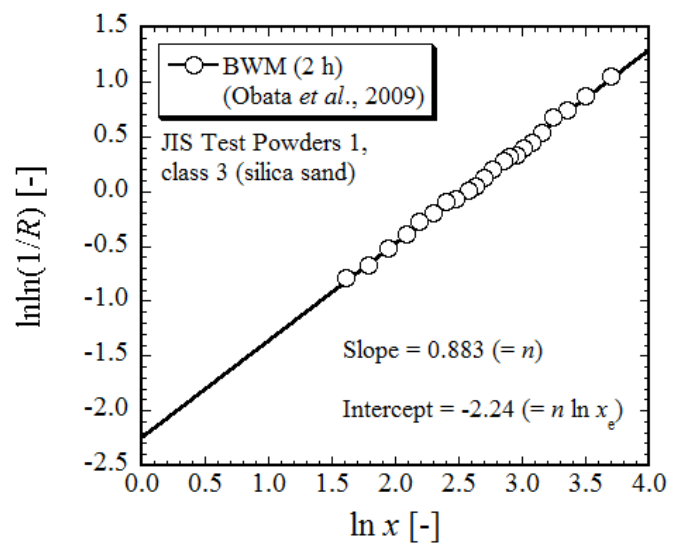

Fig. 1 Rosin-Rammler plot (JIS Test Powders 1, class 3(Silica sand))

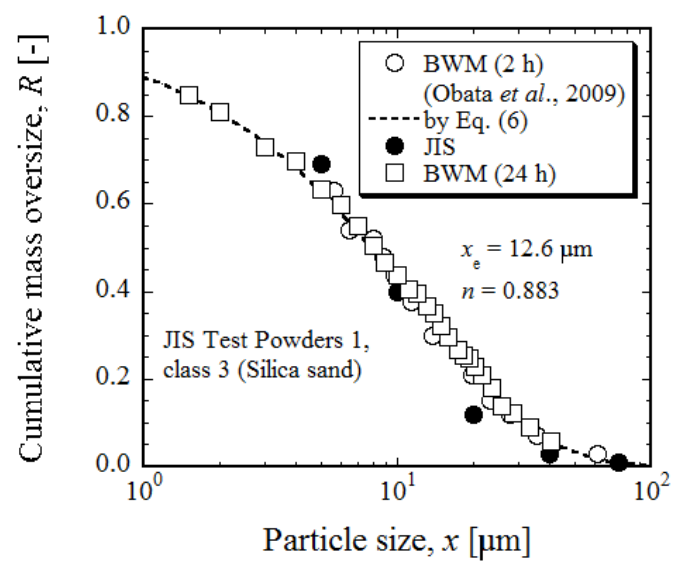

Fig. 2 Particle size distributions of JIS Test Powders 1, class 3 (Silica sand)

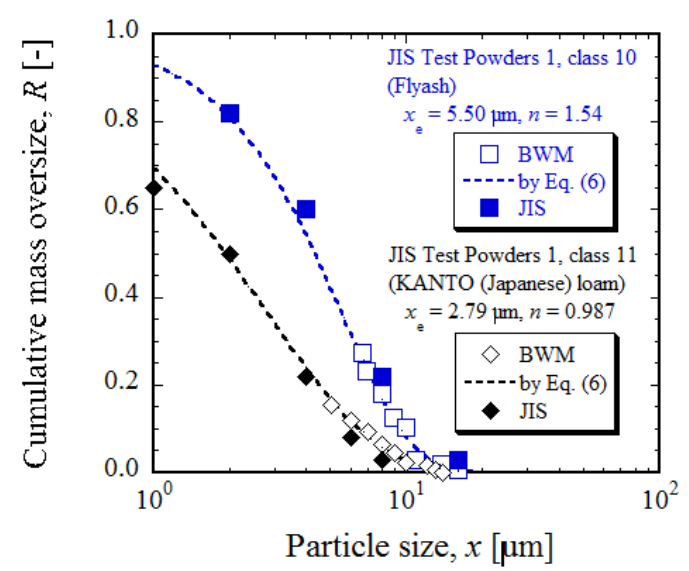

Fig. 3 Particle size distributions of JIS Test Powders 1, class 10 (Flyash) and class 11 (KANTO (Japanese) loam)

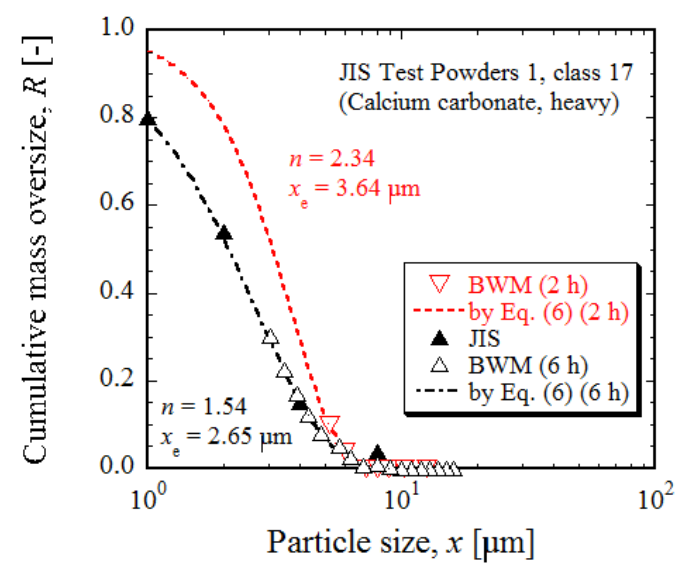

Fig. 4 Particle size distributions of JIS Test Powders 1, class 17(Calcium carbonate, heavy)

Powders 1, class 17 (Calcium carbonate, heavy), the calculation results from the Rosin-Rammler equation and the JIS standard value. The value of the cumulative mass oversize is up to about 0.10 within $2 \mathrm{~h}$. In this case, the calculated results and the JIS standard values do not agree. Because the particle size distributions can be estimated by the Rosin-Rammler equation when the value of cumulative mass oversize exceeds 0.15 , the particle size distribution within $6 \mathrm{~h}$ is measured. The triangles and dashed line in Figure 4 depict the particle size distribution and the calculated result using the Rosin-Rammler equation, respectively. The value of the cumulative mass oversize is up to about 0.30 within $6 \mathrm{~h}$. The calculated results and the JIS standard values agree well. These results demonstrate that the particle size distribution can be estimated with the Rosin-Rammler equation when the cumulative mass oversize $R$ exceeds 0.15 .

\subsection{Application for natural particles}

Figure 5 shows the particle size distributions of the 


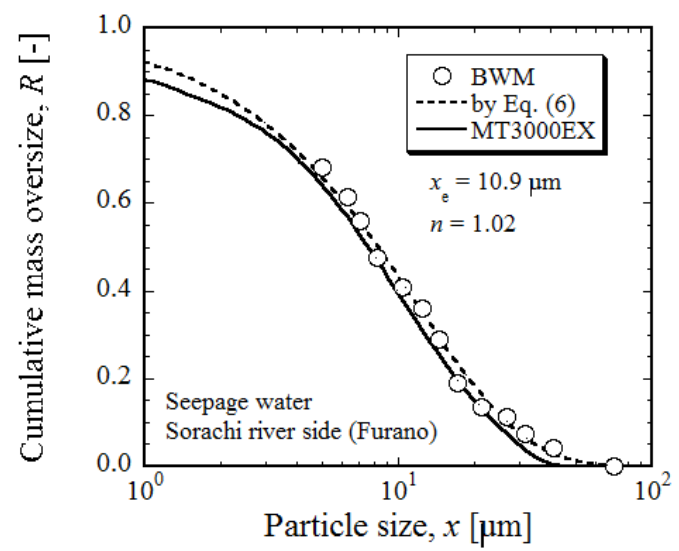

Fig. 5 Particle size distributions of suspended solids in seepage water at the construction site near the Sorachi River in Furano, Hokkaido, Japan

suspended solids in seepage water at the construction site measured over 2 h. Particle sizes from 5 to $71 \mu \mathrm{m}$ can be measured by the buoyancy weighing-bar method. The particle size distribution (dashed line) can be estimated with the Rosin-Rammler equation because the cumulative mass oversize $R$ is up to about 0.70 . Absolute size constant $x_{\mathrm{e}}$ and distribution constant $n$ are $10.9 \mu \mathrm{m}$ and 1.02, respectively. The particle size distribution measured by the laser diffraction/scattering method (solid line) and that estimated by the Rosin-Rammler equation give similar results, confirming that the buoyancy weighing-bar method can be used to estimate the particle size distribution of suspended solids in seepage water. With respect to the distribution constant $n$ in the Rosin-Rammler equation (Eqs. (6) and (7)), a log-normal distribution is appropriate for modeling the particle size distribution if $n>3$ (Miwa, 1981). While, the powder having a broad particle size distribution (e.g., ground products and dust particles) does not accord with log-normal distribution. As the $n$ values of samples used in this study are consequently less than 3 , we should apply the Rosin-Rammler equation to estimate its size distribution. At the present stage, we do not find a clear criterion for the setting value of cumulative mass oversize. Thus, the physical meaning of the value, a range in application of Rosin-Rammler equation, and their relation should be demonstrated to precisely estimate particle size distributions by using the proposed method. Further investigation is needed in order to apply the proposed method to a wide variety of samples. In this paper, however, we proposed the Rosin-Rammler equation as an example to estimate the particle size distribution. We will report the application of this method to other samples on the future article.

\section{Conclusions}

The particle size distribution is important information in the sedimentation method. Evaluating the estimation validity of the particle size distributions using the Rosin-Rammler equation has lead to the following results:

(1) The particle size distributions of JIS Test Powders 1, class 3, class 10 , and class 11 can be measured by the buoyancy weighing-bar method within $2 \mathrm{~h}$, and estimated using the Rosin-Rammler equation.
(2) The Rosin-Rammler equation cannot estimate the particle size distribution of JIS Test Powders 1, class 17 because the cumulative mass oversize is up to about 0.1 within $2 \mathrm{~h}$. However, when the cumulative mass oversize is 0.15 or more, the Rosin-Rammler equation can be used to estimate the particle size distribution.

(3) The particle size distribution of suspended solids in seepage water can be estimated using data from the buoyancy weighing-bar method.

\section{Acknowledgements}

The authors wish to thank Messrs. K. Yamamoto and Y. Inaba.

\section{Nomenclature}

$C \quad=$ particle concentration $\quad\left[\mathrm{kg} \cdot \mathrm{m}^{-3}\right]$

$f(x) \quad=$ mass frequency of the particle size $x$

g = gravitational acceleration $\left[\mathrm{m} \cdot \mathrm{s}^{-2}\right]$

$G \quad=$ apparent mass of the weighing bar $\quad[\mathrm{kg}]$

$\begin{array}{lll}G & =\text { apparent mass of the weighing bar } & {[\mathrm{kg}]} \\ G_{\mathrm{R}} & =\text { intercept of Eq.(3) } & {[\mathrm{kg}]}\end{array}$

$h \quad$ = submerged length of the weighing bar [ $\mathrm{m}$ ]

n = distribution constant [ - ]

$R \quad=$ cumulative mass oversize [ ]

$t \quad=$ time [s]

$v(x) \quad=$ settling velocity of particle size $x \quad\left[\mathrm{~m} \cdot \mathrm{s}^{-1}\right]$

$V_{\mathrm{B}} \quad=$ submerged volume of the weighing bar $\quad\left[\mathrm{m}^{3}\right]$

$W \quad$ = buoyant mass of the submerged weighing bar $\quad[\mathrm{kg}]$

$x \quad=$ particle size [

$x_{\mathrm{e}} \quad=$ absolute size constant $\quad[\mathrm{m}]$

$x_{\mathrm{i}} \quad=$ particle size of $i$ th

$\mu_{\mathrm{L}} \quad=$ viscosity of the dispersion liquid $\quad[\mathrm{Pa} \cdot \mathrm{s}]$

$\rho_{\mathrm{L}} \quad=$ density of the dispersion liquid $\quad\left[\mathrm{kg} \cdot \mathrm{m}^{-3}\right]$

$\rho_{\mathrm{B}} \quad=$ weighing bar density $\quad\left[\mathrm{kg} \cdot \mathrm{m}^{-3}\right]$

$\rho_{\mathrm{P}} \quad=$ particle density $\quad\left[\mathrm{kg} \cdot \mathrm{m}^{-3}\right]$

$\rho \mathrm{s} \quad=$ suspension density $\quad\left[\mathrm{kg} \cdot \mathrm{m}^{-3}\right]$

\section{Subscripts}

$\max \quad=$ maximum

$\min \quad=$ minimum

$0 \quad=$ initial $t=0$

$\infty \quad=$ infinity $t=\infty$

\section{Literature Cited}

Allen, T.; Particle Size Measurement, 4th ed., pp.163-164, Chapman and Hall, London, UK (1990)

Andreasen, A. H. M.; "The Grinding of Materials. Theoretical and Experimental Researches on Particle-Size Distribution Incident to the Disintegration Process," Kolloid Beihefte, 27, 349-358 (1928)

Bouyoucos, G. J.; "The Hydrometer as a New Method for the Mechanical Analysis of Soils," Soil Science, 23, 343-354 (1927)

Coulter, W. H.; "Means for Counting Particles Suspended in a Fluid", US patent No. 2656508 (1953)

Donoghue, J. K. and W. Bostock; "Technique for Particle-Size Analysis by Centrifugal Sedimentation,” Trans. Inst. Chem. Eng., 33, 72-75 (1955)

Furukawa, K., Y. Ohira, E. Obata and Y. Yoshida; "Measurements of Mineral Particle Size Distributions by a Buoyancy Weighing Method," Journal of MMIJ, 126, 577-582 (2010)

Hidaka, J. and H. Kamiya; Basic Powder Technology (Kiso Funtai Kougaku), Nikkan Kogyo Shimbun, Tokyo, Japan (2014)

JIS Z8807; Measuring Methods for Specific Gravity of Solid, pp.1-12, Japanese Standard Association, Tokyo, Japan (1976)

JIS Z8901; Test Powders and Test Particles, pp.1-23, Japanese Standard Association, Tokyo, Japan (2006)

JIS K0102; Testing Methods for Industrial Wastewater, pp.1-366, Japanese Standard Association, Tokyo, Japan (2008)

JIS A1202; Testing Methods for Density of Soil Particles, pp.1-8, Japanese Standard Association, Tokyo, Japan (2009)

JIS K8803-01; Methods for Viscosity Measurement of Liquid, pp.1-40, Japanese Standard Association, Tokyo, Japan (2011)

Minoshima, H., K. Matsushima and K. Shinohara; "Experimental Study on Size Distribution of Granules Prepared by Spray Drying: The Case of a Dispersed Slurry Containing Binder," Kagaku Kogaku Ronbunshu, 31, 102-107 (2005)

Miwa, S.; General Powder Technology (Funtai Kougaku Tsuron), Nikkan Kogyo Shimbun, Tokyo, Japan (1981)

Obata, E., Y. Ohira and M. Ohta; "New Measurement of Particle Size Distribution 
by Buoyancy Weighing-bar Method,” Powder Technol., 196, 163-168 (2009) Odén, S.; "The Size Distribution of Particles in Soils and the Experimental Methods of Obtaining Them," Soil Science, 19, 1-35 (1925)

Ohira, Y., K. Furukawa, R. Tambun, M. Shimadzu and E. Obata; "Buoyancy
Weighing-bar Method: A Particle Size Distribution Measurement Using New Settling Method,” J. Sediment. Soc. Japan, 69, 17-26 (2010)

Stober, W. and A. Fink; "Controlled Growth of Monodisperse Silica in the Micron Size Range,” J. Colloid Interface Sci., 26, 62-69 (1968) 